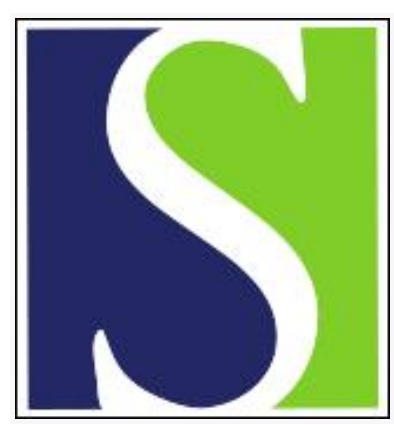

Scand J Work Environ Health 2002;28(4):211-213

https://doi.org/10.5271/sjweh.667

Issue date: Aug 2002

Static muscular load, an increasing hazard in modern information technology

by Takala E-P

Affiliation: Finnish Institute of Occupational Health, Topeliuksenkatu 41 a A, FI-00250 Helsinki, Finland.

Refers to the following texts of the Journal: 2002;28(4):215-221 1999;25 suppl 4:19-24 2002;28(1):18-24

Key terms: editorial; information technology; static muscular load

This article in PubMed: www.ncbi.nlm.nih.gov/pubmed/12199421

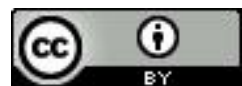




\section{Static muscular load, an increasing hazard in modern information technology}

Musculoskeletal disorders have traditionally been seen as problems associated with heavy physical work. Epidemiologic studies indicate that repetition, force, and vibration have a causal relationship with respect to work-related upper-extremity disorders (1). In physically light work these conditions have been rare. Writer's cramp has been featured in the medical literature for 200 years or so, and telegraphist's wrist also made an appearance in the early days of electrical communication. In the last few decades more attention has been paid to the increasing number of neck and upper-limb symptoms among office workers. This increase seems to go together with the new developments in information technology.

According to national statistics, the risk of a clinical diagnosis of an upper-limb disease among office workers still seems to be minimal in comparison with the traditional high-risk industries like manufacturing or food processing. In a recent study, however, more than $50 \%$ of computer users reported musculoskeletal symptoms in the neck or upper extremities during the first year on a new job (2). Most of the clinical examination findings concerning neck disorders were comprised of nonspecific limitations of movements and tenderness in the palpation of muscles.

How can physically light, computer work be hazardous? Punnett \& Bergqvist systematically analyzed 56 epidemiologic studies published on work with a video display unit before 1997 (3). Most of these studies were cross-sectional, but a trend was seen between work on a computer and musculoskeletal problems. Additional factors increasing the risk were use of spectacles, high work pace, insufficiency of rest breaks, and poor workstation ergonomics. Occupational health practitioners experience common findings; namely, workers searching for medical advice often report that their symptoms appeared after a long period of intense computer work.

Modern office work has characteristics that are similar to any work requiring precise coordination between hand and eye movements. Visual information is presented on a screen, and the information is handled by manual input devices like the keyboard or mouse. All the equipment is stable in the same position on the table and therefore requires the worker to keep the same static posture during work. A sedentary position has been preferred to standing because, in sitting, less muscular energy is needed to stabilize the upper body. Therefore it is possible to continue working for long periods without feeling fatigue that would stop the work in a static standing position or in physically heavier dynamic work. Concentration on the task prevents the worker from becoming aware of the early signals of physiological fatigue or discomfort within the body. The more interesting the task, the easier it is to forget the time and go on without breaks.

Insufficient recovery after local muscular fatigue is believed to be essential in the genesis of muscular pain in static work. According to the prevailing hypothesis, prolonged activity of single muscle fibers may cause degenerative changes in the muscles (4). Even with very low levels of static muscular contraction. it is possible for some muscle fibers to be overloaded long before the whole muscle is exhausted. During a static contraction of a muscle, new motor units are recruited to maintain the required level of muscular force and to allow recovery for the fibers that have depleted their energy. If the force development is low, the fibers with the lowest activation threshold are activated first, while the rest of the fibers may stay inactive. It has been shown that some fibers can behave like Cinderella in the fairy tale: they stay active during a long-lasting contraction while the others rest.

The physiological pathway from muscular fatigue to pain is still controversial (4). It is probable that pain results through several different mechanisms that interact. Several reflex mechanisms can 
contribute to the vicious circles and increase both tonic muscular activity and pain. Local biochemical and inflammation reactions, in addition to the reflexes affecting blood circulation, can make the interactions extremely complex.

Fatigue due to static contraction highly depends on the activity level of the muscle. The higher the activity with respect to muscle capacity, the sooner fatigue appears. In computer work, muscular activity of the neck and shoulder muscles resists the gravity acting on the forward leaning head while the worker gazes at the screen. The kinetic chain formed of the bones and joints of the upper limb has to be stabilized by the muscles to enable exact movements of the fingers and hands. If there is no mechanical support for the forearms, the shoulder muscles must hold the weight of the whole upper extremity, and this action further increases muscle tension.

In this issue of the Scandinavian Journal of Work, Environment \& Health, the paper of Laursen et al (5) demonstrates that mental stressors increase muscular activity in computer work. In neck muscles, greater activity was found during the use of the mouse than with the keyboard. The authors credibly explain that the use of the mouse introduces higher visual demands, and therefore a more accurate posture of the head is needed. These results confirm previous findings (6) well. Physiologically, the increased muscular activity with mental tasks probably presents the same phenomena as other autonomic reactions against stress, by preparing the individual to fight or flee (4). Individual factors seem to affect highly the strength of such reactions (7).

The basic function of the musculoskeletal system is movement. The "work" of our ancestors was almost continuous movement in search of nourishment. Sometimes short periods of heavy physical exertion were necessary during fighting or fleeing. These exertions were followed by periods of recovery. The time spent in static, stationary body positions during each day would have amounted to minutes rather than hours. Work paced by the clock has constrained the human body to static postures or repeated physical exertions during some tens of generations. There has not, however, been any biological evolutionary adaptation to the demands of modern work. Our body is structurally and functionally similar to that of our ancestors. Therefore, the long-lasting static postures of work or leisure time seem to be against our biological nature. The organization and attitudes in modern worklife towards longer and longer workhours, time pressure, and other psychosocial stress factors obviously reduce the individual's opportunities for recovery.

Technological innovations may change the human-computer interface so that the person's hand-eye coordination will not necessarily be constrained by a physically stable posture. Still contemporary technology with visual display units will predominate for years. Enough knowledge already exists for preventive measures against the hazards of static load in work with modern technology, as well as that found in traditional work. Biomechanical load can be reduced by ergonomic measures of the physical workstation (8). Without simultaneous changes in the temporal aspects of tasks, changes in the human computer interface - especially visual usability — and adaptation of psychosocial workload, the benefits of physical ergonomic changes in the prevention of disorders may remain scant. The effectiveness of different interventions has to be evaluated in controlled randomized trials. The other challenges of future research remain in defining safety limits for the duration of static work periods with respect to recovery time and means.

\section{References}

1. National Research Council and Institute of Medicine, Panel on Musculoskeletal Disorders and the Workplace, Commission on Behavioral and Social Sciences and Education. Musculoskeletal disorders and the workplace: low back and upper extremities. Washington (DC): National Academy Press, 2001.

2. Gerr F, Marcus M, Ensor C, Kleinbaum D, Cohen S, Edwards A, et al. A prospective study of computer users, I: study design and incidence of musculoskeletal symptoms and disorders. Am J Ind Med 2002;41(4):221-35. 
3. Punnett L, Bergqvist U. Visual display unit work and upper extremity musculoskeletal disorders. Solna (Sweden): Arbetslivsinstitutet, 1997:1-161. Arbete och hälsa, no 1997;16.

4. Sjøgaard G, Lundberg U, Kadefors R. The role of muscle activity and mental load in the development of pain and degenerative processes at the muscle cell level during computer work. Eur J Appl Physiol 2000;83(2-3):99-105.

5. Laursen B, Jensen B, Garde A, Jørgensen A. Effect of mental and physical demands on muscular activity during the use of a computer mouse and a keyboard. Scand J Work Environ Health 2002;28(4)215-21.

6. Wærsted M. Human muscle activity related to non-biomechanical factors in the workplace. Eur J Appl Physiol 2000;83 (2-3):151-158.

7. Westgaard R. Effects of physical and mental stressors on muscle pain. Scand J Work Environ Health 1999;25 suppl 4:19-24.

8. Ketola R, Toivonen R, Häkkänen M, Luukkonen R, Takala E-P, Viikari-Juntura E. Effects of ergonomic intervention in work with video display units. Scand J Work Environ Health 2002;28(1):18-24.

Esa-Pekka Takala, DMedSc

Finnish Institute of Occupational Health

Topeliuksenkatu 41 a $\mathrm{A}$

FIN-00250 Helsinki, Finland 
